\title{
The Scrambling Index of Two-colored Wielandt Digraph
}

\author{
Mulyono, Saib Suwilo* \\ Department of Mathematics, University of Sumatera Utara, Medan 20155, Indonesia \\ *Corresponding Author: saib@usu.ac.id
}

Copyright (c) 2014 Horizon Research Publishing All rights reserved.

\begin{abstract}
A digraph is primitive provided there is a positive integer $k$ such that for each pair of vertices $u$ and $v$ there exist walks of length $k$ from $u$ to $v$ and from $v$ to $u$. The scrambling index of a primitive digraph $D$ is the smallest positive integer $k$ such that for each pair of vertices $u$ and $v$ in $D$ there is a vertex $w$ such that there exist walks of length $k$ from $u$ to $w$ and from $v$ to $w$. A two-colored digraph is a digraph each of whose arc is colored by red or blue. In this paper we generalize the notion of scrambling index of a primitive digraph to that of two-colored digraph. We define the scrambling index of a two-colored digraph $D^{(2)}$ to be the smallest positive integer $h+\ell$ over all pairs of nonnegative integers $(h, \ell)$ such that for each pair of distinct vertices $u$ and $v$ there is a vertex $w$ with the property that there are walks form $u$ to $w$ and from $v$ to $w$ consisting of $h$ red arcs and $\ell$ blue arcs. For two-colored Wielandt digraph on $n \geq 4$ vertices we show the scrambling index lies on the interval $\left[n^{2}-3 n+3, n^{2}-2 n+2\right]$.
\end{abstract}

Keywords Two-colored digraph, Primitive digraph, Scrambling Index, Wielandt digraph

\section{Introduction}

By a nonnegative integer vector $\mathbf{x} \geq 0$ we meant a vector each of whose entry is a nonnegative integer. Therefore, the notion $\mathbf{z} \geq \mathbf{x}$ means that $\mathbf{z}-\mathbf{x} \geq 0$.

Let $D$ be a digraph. A walk of length $k$ from $u$ to $v$ is a sequence of arcs of the form $u=v_{0} \rightarrow v_{1}, v_{1} \rightarrow$ $v_{2}, \ldots, v_{k-1} \rightarrow v_{k}=v$. We use the notation $u \stackrel{k}{\rightarrow} v$ walk to represent a walk of length $k$ from $u$ to $v$. A $u \rightarrow v$ path is a walk with distinct vertices except possibly $u=v$. A cycle is a $u \rightarrow v$ path with $u=v$. A digraph $D$ is strongly connected if for each pair of vertices $u$ and $v$ there is a $u \rightarrow v$ walk and a $v \rightarrow u$ walk. A strongly connected digraph $D$ is primitive provided there is a positive integer $k$ such that for each pair of vertices $u$ and $v$ there exist a $u \stackrel{k}{\rightarrow} v$ walk and a $v \stackrel{k}{\rightarrow} u$ walk. The smallest of such positive integer $k$ is the exponent of $D$ and is denoted by $\exp (D)$. It is a well known result that for a primitive digraph on $n$ vertices, see [4], the $\exp (D) \leq(n-1)^{2}+1$. The upper bound is achieved the Wielandt digraph $W_{n}$ on $n$ vertices that is a digraph consists of a Hamiltonian cycle $v_{1} \rightarrow v_{2} \rightarrow \cdots \rightarrow v_{n} \rightarrow v_{1}$ and the arc $v_{n-1} \rightarrow v_{1}$ as in Figure 1 .

The notion of scrambling index of a primitive digraph was first introduced by Akelbek and Kirkland [1, 2]. They define the scrambling index of a primitive digraph $D$ to be the smallest positive integer $k$ such that for every pair of vertices $u$ and $v$ in $D$ there exists a vertex $w$ in $D$ such that there is a $u \stackrel{k}{\rightarrow} w$ walk and a $v \stackrel{k}{\rightarrow} w$ walk. The scrambling index of a primitive digraph is denoted by $k(D)$. Their results, see [2], show that primitive digraph with largest scrambling index is achieved by the Wielandt digraph.

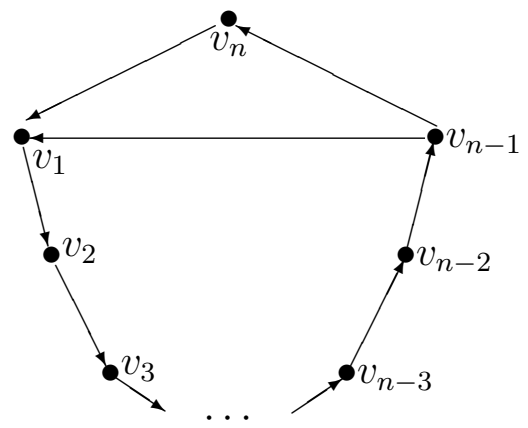

Figure 1. The Wielandt Digraph $W_{n}$

A two-colored digraph is a digraph each of whose arc is colored by red or blue. For nonnegative integers $h$ and $\ell$, an $(h, \ell)$-walk in a two-colored digraph is a walk consisting of $h$ red arcs and $\ell$ blue arcs. An $(h, \ell)$-walk from $u$ to $v$ is denoted by $u \stackrel{(h, \ell)}{\longrightarrow} v$. For a walk $W$ in $D^{(2)}$, we denote $r(W)$ and $b(W)$ respectively to be the number of red arcs and blue arcs of $W$. The vector $\left[\begin{array}{l}r(W) \\ b(W)\end{array}\right]$ is the composition of $W$. A strongly connected two-colored digraph $D^{(2)}$ is primitive provided that there are nonnegative integers $h$ and $\ell$ such that for each pair of vertices $u$ and $v$ in $D^{(2)}$ there exist a $u \stackrel{(h, \ell)}{\longrightarrow} v$ walk and a $v \stackrel{(h, \ell)}{\longrightarrow} u$ walk.

Let $D^{(2)}$ be a two-colored digraph and let $C=$ $\left\{C_{1}, C_{2}, \ldots, C_{q}\right\}$ be the set of all cycles in $D^{(2)}$. Define the cycle matrix $M$ of $D^{(2)}$ to be the matrix

$$
M=\left[\begin{array}{llll}
r\left(C_{1}\right) & r\left(C_{2}\right) & \cdots & r\left(C_{q}\right) \\
b\left(C_{1}\right) & b\left(C_{2}\right) & \cdots & b\left(C_{q}\right)
\end{array}\right] .
$$

That is $M$ is the matrix such that the $i$ th column of $M$ is the composition of the cycle $C_{i}, i=1,2, \ldots, q$. The content of 
the 2 by $q$ matrix $M$ is defined to be 0 if the rank of $M$ is less than 2 and the greatest common divisor of the determinants of the 2 by 2 submatrices of $M$, otherwise. The following theorem presents an algebraic characterization for a primitive two-colored digraph.

Theorem 1.1 [5] Let $D^{(2)}$ be a two-colored digraph with at least one arc of each color. The two-colored digraph $D^{(2)}$ is primitive if and only if the content of its cycle matrix is 1 .

We generalize the notion of scrambling index of a primitive digraph to that of scrambling index of a primitive twocolored digraph. For a primitive two-colored digraph $D^{(2)}$ we define the scrambling index of $D^{(2)}$ to be the smallest positive integer $h+\ell$ over all nonnegative integers $h$ and $\ell$ such that for every pair of vertices $u$ and $v$ in $D^{(2)}$ there is a vertex $w$ with the property that there is a $u \stackrel{(h, \ell)}{\longrightarrow} w$ walk and a $v \stackrel{(h, \ell)}{\longrightarrow} w$ walk. The scrambling index of $D^{(2)}$ is denoted by $k\left(D^{(2)}\right)$.

Ananichev, Gusev, and Volkov [3] have used primitive digraphs with large exponents in attempt to find slowly synchronizing automata. Such primitive digraphs consist of cycles with two distinct lengths. An automaton on two input letters over a finite states is synchronizing if there exists a word, called a reset word, of finite length that brings all states to a particular state. Černý's conjecture states that for an automaton on two input letters $\mathcal{A}$ with $n$ states, the length of a reset word is no more than $(n-1)^{2}$. This is close to the exponent of a Wielandt digraph on $n$ vertices which is $(n-1)^{2}+1$.

Let $\mathcal{A}$ be a synchronizing automaton on two input letters and let $D^{(2)}$ be a two-colored digraph representation of $\mathcal{A}$. An automaton $\mathcal{A}$ is synchronizing with reset word of length $h+\ell$ if there exists a vertex $u$ in $D^{(2)}$ such that for each vertex $v$ in $D^{(2)}$ there is a $v \stackrel{(h, \ell)}{\longrightarrow} u$ walk, moreover the order of appearance of red and blue arcs in each $v \rightarrow u$ walk are the same. Thus the scrambling index of a two-colored digraph may be used as a lower bound for the length of a reset word for a synchronizing automaton with two input letters.

In this paper, we discuss the scrambling index of twocolored Wielandt digraphs $W_{n}^{(2)}$ that is a two-colored digraph obtained by coloring each arc of the Wielandt digraph $W_{n}$ with either red or blue. In Section 2, we discuss a way to determine a lower and an upper bound for scrambling index of two-colored digraph consisting two cycles. In Section 3 we discuss the scrambling index of two-colored Wielandt digraph.

\section{Lower and Upper Bound}

In this section, we discuss a way in setting up lower and upper bound for scrambling index of primitive two-colored digraph, especially those that consist of two cycles.

We first note that every walk in a two-colored digraph can be decomposed into a path and some cycles. This implies for every $u \stackrel{(h, \ell)}{\longrightarrow} v$ walk we have the following relationship

$$
\begin{aligned}
{\left[\begin{array}{l}
h \\
\ell
\end{array}\right]=} & {\left[\begin{array}{l}
r\left(p_{u v}\right) \\
b\left(p_{u v}\right)
\end{array}\right]+z_{1}\left[\begin{array}{l}
r\left(C_{1}\right) \\
b\left(C_{1}\right)
\end{array}\right] } \\
& +z_{2}\left[\begin{array}{l}
r\left(C_{2}\right) \\
b\left(C_{2}\right)
\end{array}\right]+\cdots+z_{q}\left[\begin{array}{l}
r\left(C_{q}\right) \\
b\left(C_{q}\right)
\end{array}\right] \\
= & {\left[\begin{array}{l}
r\left(p_{u v}\right) \\
b\left(p_{u v}\right)
\end{array}\right]+M \mathbf{z} }
\end{aligned}
$$

for some path $p_{u v}$ from $u$ to $v$ and some nonnegative integer vector $\mathbf{z}$.

The following proposition will be useful in order to determine an upper bound for scrambling index.

Proposition 2.1 Let $D^{(2)}$ be a primitive two-colored digraph consisting of two cycles $C_{1}$ and $C_{2}$. Suppose $v$ is a vertex that belongs to both cycles. If for some positive integers $h$ and $\ell$, there is a path $p_{u, v}$ from $u$ to $v$ such that the system

$$
M \mathbf{z}+\left[\begin{array}{c}
r\left(p_{u, v}\right) \\
b\left(p_{u, v}\right)
\end{array}\right]=\left[\begin{array}{c}
h \\
\ell
\end{array}\right]
$$

has nonnegative integer solution, then there is an $(h, \ell)$-walk from $u$ to $v$.

Proof. Assume that the solution to the system (1) is $\mathbf{z}=$ $\left(z_{1}, z_{2}\right)^{T}$. We consider four cases.

If $z_{1}>0$ and $z_{2}>0$, then the walk that starts at $u$, moves to $v$ along the $\left(r\left(p_{u, v}\right), b\left(p_{u, v}\right)\right)$-path $p_{u, v}$ and finally moves $z_{1}$ and $z_{2}$ times around the cycles $C_{1}$ and $C_{2}$, respectively, and back at $v$ is an $(h, \ell)$-walk from $u$ to $v$.

If $z_{1}=0$ and $z_{2}>0$, then the walk that starts at $u$, moves to $v$ along the $\left(r\left(p_{u, v}\right), b\left(p_{u, v}\right)\right)$-path $p_{u, v}$ and finally moves $z_{2}$ times around the cycle $C_{2}$ and back at $v$ is an $(h, \ell)$-walk from $u$ to $v$. Similarly if $z_{1}>0$ and $z_{2}=0$, then the walk that starts at $u$, moves to $v$ along the $\left(r\left(p_{u, v}\right), b\left(p_{u, v}\right)\right)$-path $p_{u, v}$ and finally moves $z_{1}$ times around the cycle $C_{1}$ and back at $v$ is an $(h, \ell)$-walk from $u$ to $v$.

Finally, if $z_{1}=z_{2}=0$, then the $\left(r\left(p_{u, v}\right), b\left(p_{u, v}\right)\right)$-path $p_{u, v}$ from $u$ to $v$ is an $(h, \ell)$-walk.

We next discuss a way in setting up a lower bound for the scrambling index. Let $u$ and $v$ be two different vertices in a primitive two-colored digraph $D^{(2)}$. For a vertex $w$ in $D^{(2)}$, the local scrambling index of $u$ and $v$ at the vertex $w$, $k_{u, v}(w)$, is the smallest positive integer $h+\ell$ over all pairs of nonnegative integers $h$ and $\ell$ such that there are $u \stackrel{(h, \ell)}{\longrightarrow} w$ and $v \stackrel{(h, \ell)}{\longrightarrow} w$ walks. The local scrambling index of vertices $u$ and $v$ in $D^{(2)}$, denoted $k_{u, v}\left(D^{(2)}\right)$, is defined by

$$
k_{u, v}\left(D^{(2)}\right)=\min _{w}\left\{k_{u, v}(w)\right\}
$$

From the definition of scrambling index we have

$$
\max _{u, v \in V\left(D^{(2)}\right)}\left\{k_{u, v}\left(D^{(2)}\right)\right\} \leq k\left(D^{(2)}\right) .
$$

Let $D^{(2)}$ be a primitive two-colored digraph consisting of two cycles and let $u$ and $v$ be two distinct vertices in $D^{(2)}$. For some vertex $w$ suppose that $k_{u, v}(w)$ is obtained by an $(h, \ell)$-walk. We have the following result that will be useful in finding a lower bound for $k_{u, v}\left(D^{(2)}\right)$ and hence for the scrambling index.

Lemma 2.2 Let $D^{(2)}$ be a primitive two-colored digraph consisting of two cycles $C_{1}$ and $C_{2}$ with cycle matrix $M=\left[\begin{array}{ll}r\left(C_{1}\right) & r\left(C_{2}\right) \\ b\left(C_{1}\right) & b\left(C_{2}\right)\end{array}\right]$, and let $u$ and $v$ be any two distinct vertices in $D^{(2)}$. Suppose there is a vertex $w$ such that there is a $u \stackrel{(h, \ell)}{\longrightarrow} w$ walk and $v \stackrel{(h, \ell)}{\longrightarrow} w$ walk. If $q_{1}$ and $q_{2}$ are integers such that $\left[\begin{array}{l}h \\ \ell\end{array}\right]=M\left[\begin{array}{l}q_{1} \\ q_{2}\end{array}\right]$, then $\left[\begin{array}{l}q_{1} \\ q_{2}\end{array}\right] \geq M^{-1}\left[\begin{array}{l}r\left(p_{u w}\right) \\ b\left(p_{u w}\right)\end{array}\right]$ for some path $p_{u w}$, and $\left[\begin{array}{l}q_{1} \\ q_{2}\end{array}\right] \geq M^{-1}\left[\begin{array}{l}r\left(p_{v w}\right) \\ b\left(p_{v w}\right)\end{array}\right]$ for some path $p_{v w}$. 
Proof. Since $D^{(2)}$ is primitive, then by Theorem 1.1 we have $\operatorname{det}(M)= \pm 1$. Without loss of generality we assume that $\operatorname{det}(M)=1$. Since every walk can be decomposed into a path and some cycles, then

$$
\left[\begin{array}{c}
h \\
\ell
\end{array}\right]=\left[\begin{array}{c}
r\left(p_{u w}\right) \\
b\left(p_{u w}\right)
\end{array}\right]+M \mathbf{z}
$$

for some path $p_{u w}$ from $u$ to $w$ and some nonnegative integer vector z. Comparing (3) and $\left[\begin{array}{l}h \\ \ell\end{array}\right]=M\left[\begin{array}{l}q_{1} \\ q_{2}\end{array}\right]$, we have

$$
\mathbf{z}=\left[\begin{array}{l}
q_{1} \\
q_{2}
\end{array}\right]-M^{-1}\left[\begin{array}{r}
r\left(p_{u w}\right) \\
b\left(p_{u w}\right)
\end{array}\right] \geq 0
$$

Hence

$$
\left[\begin{array}{l}
q_{1} \\
q_{2}
\end{array}\right] \geq M^{-1}\left[\begin{array}{l}
r\left(p_{u w}\right) \\
b\left(p_{u w}\right)
\end{array}\right]
$$

for some path $p_{u w}$. Similarly

$$
\left[\begin{array}{l}
q_{1} \\
q_{2}
\end{array}\right] \geq M^{-1}\left[\begin{array}{l}
r\left(p_{v w}\right) \\
b\left(p_{v w}\right)
\end{array}\right]
$$

for some path $p_{v w}$.

We note from Lemma 2.2 that

$$
\begin{aligned}
{\left[\begin{array}{l}
q_{1} \\
q_{2}
\end{array}\right] } & =M^{-1}\left[\begin{array}{l}
r\left(p_{u w}\right) \\
b\left(p_{u w}\right)
\end{array}\right] \\
& =\left[\begin{array}{l}
b\left(C_{2}\right) r\left(p_{u w}\right)-r\left(C_{2}\right) b\left(p_{u w}\right) \\
r\left(C_{1}\right) b\left(p_{u w}\right)-b\left(C_{1}\right) r\left(p_{u w}\right)
\end{array}\right] .
\end{aligned}
$$

Hence we have

$$
q_{1} \geq b\left(C_{2}\right) r\left(p_{u w}\right)-r\left(C_{2}\right) b\left(p_{u w}\right)
$$

for some path $p_{u w}$ from $u$ to $w$. Similarly, we have

$$
q_{2} \geq r\left(C_{1}\right) b\left(p_{v w}\right)-b\left(C_{1}\right) r\left(p_{v w}\right)
$$

for some path $p_{v w}$ from $v$ to $w$. Thus

$$
\left[\begin{array}{c}
h \\
\ell
\end{array}\right] \geq M\left[\begin{array}{l}
b\left(C_{2}\right) r\left(p_{u w}\right)-r\left(C_{2}\right) b\left(p_{u w}\right) \\
r\left(C_{1}\right) b\left(p_{v w}\right)-b\left(C_{1}\right) r\left(p_{v w}\right)
\end{array}\right]
$$

for some paths $p_{u w}$ and $p_{v w}$.

\section{Main Results}

In this section we present formulae for scrambling index of two-colored Wieland digraph. We first present primitivity condition for two-colored Wielandt digraph and then discuss formulae their scrambling index.

We note that the Wielandt digraph consists of two cycles. They are the $n$-cycle $v_{1} \rightarrow v_{2} \rightarrow \cdots \rightarrow v_{n} \rightarrow v_{1}$ and the $(n-1)$-cycle $v_{1} \rightarrow v_{2} \rightarrow \cdots \rightarrow v_{n-1} \rightarrow v_{1}$. As a consequence of Theorem 1.1 we have the following characterization for primitivity of a two-colored Wielandt digraph.

Lemma 3.1 [6] A two-colored Wielandt digraph $W_{n}^{(2)}$ on $n$ vertices is primitive if and only if its cycle matrix $M=$ $\left[\begin{array}{ll}r\left(C_{1}\right) & r\left(C_{2}\right) \\ b\left(C_{1}\right) & b\left(C_{2}\right)\end{array}\right]=\left[\begin{array}{ll}n-1 & n-2 \\ 1 & 1\end{array}\right]$
Lemma 3.1 implies that a primitive two-colored Wielandt digraph has at most two blue arcs. Moreover, every cycle contains exactly one blue arc. We determine the scrambling index of $W_{n}^{(2)}$ based on how many blue arcs $W_{n}^{(2)}$ has. If $W_{n}^{(2)}$ has only one blue arcs, then the blue arc must lie on the $v_{1} \rightarrow v_{n-2}$ path. So the blue arc of $W_{n}^{(2)}$ must be of the form $v_{a} \rightarrow v_{a+1}$ where $1 \leq a \leq n-2$. If $w_{n}^{(2)}$ has two blue arcs, then one of them must lie on the cycle $C_{2}$ but not on $C_{1}$ and the other must lie on $C_{1}$ but not on $C_{2}$. This implies the two blue arcs either have the same terminal vertex or have the same initial vertex.

We first discuss the case where $W_{n}^{(2)}$ has only one blue arc and then discuss the case where $W_{n}^{(2)}$ has two blue arcs.

Theorem 3.2 Let $W_{n}^{(2)}$ be a two colored Wielandt digraph on $n \geq 4$ vertices. If $W_{n}^{(2)}$ has only one blue arc $v_{a} \rightarrow v_{a+1}$, where $1 \leq a \leq n-2$, then $k\left(W_{n}^{(2)}\right)=n^{2}-2 n+1-a$.

Proof. We show that $k\left(W_{n}^{(2)}\right) \geq n^{2}-2 n+1-a$. This is done by showing that $k_{v_{a}, v_{a+1}}\left(W_{n}^{(2)}\right) \geq n^{2}-2 n+1-a$. We assume that there are $v_{a} \stackrel{(h, \ell)}{\longrightarrow} w$ and $v_{a+1} \stackrel{(h, \ell)}{\longrightarrow} w$ walks for some vertex $w \in W_{n}^{(2)}$. We present a lower bound for $k_{v_{a}, v_{a+1}}(w)$ and consider two cases depending on the position of the vertex $w$.

Case 1. The vertex $w=v_{t}$ where $1 \leq t \leq a$

Notice that there are two paths $p_{a+1, t}$ from $v_{a+1}$ to $v_{t}$. They are an $(n-2+t-a, 0)$-path and an $(n-1+t-a)$-path. Considering the $(n-2+t-a, 0)$-path and (4) we have

$$
\begin{aligned}
q_{1} & \geq b\left(C_{2}\right) r\left(p_{a+1, t}\right)-r\left(C_{2}\right) b\left(p_{a+1, t}\right) \\
& =(1)(n-2+t-a)-(n-2)(0)=n-2+t-a .
\end{aligned}
$$

Considering the $(n-1+t-a, 0)$-path and (4) we have

$$
\begin{aligned}
q_{1} & \geq b\left(C_{2}\right) r\left(p_{a+1, t}\right)-r\left(C_{2}\right) b\left(p_{a+1, t}\right) \\
& =(1)(n-1+t-a)-(n-2)(0)=n-1+t-a .
\end{aligned}
$$

Therefore we conclude that $q_{1} \geq n-2+t-a$.

There are two paths $p_{a, t}$ from $v_{a}$ to $v_{t}$. They are an $(n-$ $2+t-a, 1)$-path and an $(n-1+t-a, 1)$-path. Considering the $(n-2+t-a, 1)$-path and (5) we have

$$
\begin{aligned}
q_{2} & \geq r\left(C_{1}\right) b\left(p_{a, t}\right)-b\left(C_{1}\right) r\left(p_{a, t}\right) \\
& =(n-1)(1)-(1)(n-2+t-a)=a-t+1
\end{aligned}
$$

Considering the $(n-1+t-a, 1)$-path and (5) we have

$$
\begin{aligned}
q_{2} & \geq r\left(C_{1}\right) b\left(p_{a, t}\right)-b\left(C_{1}\right) r\left(p_{a, t}\right) \\
& =(n-1)(1)-(1)(n-1+t-a)=a-t .
\end{aligned}
$$

Therefore, we conclude that $q_{2} \geq a-t$.

Now by Lemma 2.2 we have

$$
\begin{aligned}
{\left[\begin{array}{l}
h \\
\ell
\end{array}\right] } & =M\left[\begin{array}{l}
q_{1} \\
q_{2}
\end{array}\right] \geq M\left[\begin{array}{l}
n-2+t-a \\
a-t
\end{array}\right] \\
& =\left[\begin{array}{l}
n^{2}-3 n+2+t-a \\
n-2
\end{array}\right],
\end{aligned}
$$

and hence

$$
k_{v_{a}, v_{a+1}}\left(v_{t}\right) \geq n^{2}-3 n+t-a
$$


for all $1 \leq t \leq a$.

Case 2. The vertex $w=v_{t}$ where $a+1 \leq t \leq n$

There is a unique path $p_{a+1, t}$ from $v_{a+1}$ to $v_{t}$ which is a $(t-a-1,0)$-path. Using this path and (4) we have

$$
\begin{aligned}
q_{1} & \geq b\left(C_{2}\right) r\left(p_{a+1, t}\right)-r\left(C_{2}\right) b\left(p_{a+1, t}\right) \\
& =(1)(t-a-1)-(n-2)(0)=t-a-1 .
\end{aligned}
$$

There is a unique path $p_{a, t}$ from $v_{a}$ to $v_{t}$ which is a $(t-a-$ 1,1)-path. Using this path and (5) we have

$$
\begin{aligned}
q_{2} & \geq r\left(C_{1}\right) b\left(p_{a, t}\right)-b\left(C_{1}\right) r\left(p_{a, t}\right) \\
& =(n-1)(1)-(1)(t-a-1)=n-t+a .
\end{aligned}
$$

By Lemma 2.2 we find that

$$
\begin{aligned}
{\left[\begin{array}{l}
h \\
\ell
\end{array}\right] } & =M\left[\begin{array}{l}
q_{1} \\
q_{2}
\end{array}\right] \geq M\left[\begin{array}{l}
t-a-1 \\
n-t+a
\end{array}\right] \\
& =\left[\begin{array}{l}
n^{2}-3 n+1+t-a \\
n-1
\end{array}\right]
\end{aligned}
$$

and hence

$$
k_{v_{a}, v_{a+1}}\left(v_{t}\right) \geq n^{2}-3 n+t-a
$$

for all $a+1 \leq t \leq n$.

From (6) and (7) we conclude that $k_{v_{a}, v_{a+1}}\left(W_{n}^{(2)}\right) \geq n^{2}-$ $2 n+1-a$ and by (2) we have $k\left(W_{n}^{(2)}\right) \geq n^{2}-2 n+1-a$.

It remains to show that $k\left(W_{n}^{(2)}\right) \leq n^{2}-2 n+1-a$. For each vertex $v_{t}, t=1,2, \ldots, n$, we show that there is $v_{t} \stackrel{(h, \ell)}{\longrightarrow} v_{1}$ with

$$
\left[\begin{array}{l}
h \\
\ell
\end{array}\right]=\left[\begin{array}{l}
n^{2}-3 n+3-a \\
n-2
\end{array}\right] .
$$

By Proposition 2.1 it suffices to show that the system

$$
M \mathbf{z}+\left[\begin{array}{l}
r\left(p_{t, 1}\right) \\
b\left(p_{t, 1}\right)
\end{array}\right]=\left[\begin{array}{l}
n^{2}-3 n+3-a \\
n-2
\end{array}\right]
$$

has nonnegative integer solution for some path $p_{t, 1}$ from $v_{t}$ to $v_{1}$.

The solution to the system (8) is the integer vector

$$
\mathbf{z}=\left[\begin{array}{l}
(n-1-a)+(n-2) b\left(p_{t, 1}\right)-r\left(p_{t, 1}\right) \\
a-1+r\left(p_{t, 1}\right)+b\left(p_{t, 1}\right)-b\left(p_{t, 1}\right) n
\end{array}\right]
$$

If $1 \leq t \leq a$, then there is an $(n-t, 1)$-path $p_{t, 1}$ from $v_{t}$ to $v_{1}$. Using this path we have that $z_{1}=n-3+t-a$ and $z_{2}=a-t$. Since $t \geq 1$ and $a \leq n-2$ we have $z_{1} \geq 0$ and since $t \leq a$ we have $z_{2} \geq 0$.

If $a+1 \leq t \leq n$, then there is an $(n-t, 0)$-path $p_{t, 1}$ from $v_{t}$ to $v_{1}$. Using this path we have that $z_{1}=t-(a+1)$ and $z_{2}=n-t+a-1$. Since $t \geq a+1$ we have $z_{1} \geq 0$ and since $t \leq n$ and $a \geq 1$ we have $z_{2} \geq 0$.

Thus for each $t=1,2, \ldots, n$, there is a path $p_{t 1}$ from $v_{t}$ to $v_{1}$ such that the system (8) has nonnegative integer solution. By Proposition 2.1 for each vertex $v_{t}, t=1,2, \ldots, n$, there is an $(h, \ell)$-walk from $v_{t}$ to $v_{1}$ with $h=n^{2}-3 n+3-a$ and $\ell=n-2$. We now can conclude that for each pair of distinct vertices $v_{i}$ and $v_{j}$ in $W_{n}^{(2)}$, there is vertex $v_{1}$ with the property that there are $v_{i} \stackrel{(h, \ell)}{\longrightarrow} v_{1}$ walk and $v_{j} \stackrel{(h, \ell)}{\longrightarrow} v_{1}$ walk with

$$
\left[\begin{array}{l}
h \\
\ell
\end{array}\right]=\left[\begin{array}{l}
n^{2}-3 n+3-a \\
n-2
\end{array}\right] .
$$

This implies $k\left(W_{n}^{(2)}\right) \leq n^{2}-2 n+1-a$.

We next discuss the scrambling index of primitive twocolored Wielandt digraph that contains two blue arcs. We first discuss the case where the two blue arcs have the same terminal vertex.

Theorem 3.3 Let $W_{n}^{(2)}$ be a two colored Wielandt digraph on $n \geq 4$ vertices. If $W_{n}^{(2)}$ has two blue arcs $v_{n-1} \rightarrow v_{1}$ and $v_{n} \rightarrow v_{1}$, then $k\left(W_{n}^{(2)}\right)=n^{2}-2 n+1$.

Proof. We first show that $k\left(W_{n}^{(2)}\right) \geq n^{2}-2 n+1$. It suffices to show that $k_{v_{n}, v_{1}}\left(W_{n}^{(2)}\right) \geq n^{2}-2 n+1$. We assume there are $v_{n} \stackrel{(h, \ell)}{\longrightarrow} w$ and $v_{1} \stackrel{(h, \ell)}{\longrightarrow} w$ walks for some vertex $w$ in $W_{n}^{(2)}$. We set up a lower bound for $k_{v_{n}, v_{1}}(w)$.

Notice that for each $t=1,2, \ldots, n$, there is a unique path $p_{1, t}$ from $v_{1}$ to $v_{t}$ which is a $(t-1,0)$-path and there is a unique path $p_{n, t}$ from $v_{n}$ to $v_{t}$ which is a $(t-1,1)$-path. Using the $(t-1,0)$-path from $v_{1}$ to $v_{t}$ and (4) we have

$$
\begin{aligned}
q_{1} & \geq b\left(C_{2}\right) r\left(p_{1, t}\right)-r\left(C_{2}\right) b\left(p_{1, t}\right) \\
& =(1)(t-1)-(n-2)(0)=t-1
\end{aligned}
$$

Using the $(t-1,1)$-path from $v_{n}$ to $v_{t}$ and (5) we have

$$
\begin{aligned}
q_{2} & \geq r\left(C_{1}\right) b\left(p_{n, t}\right)-b\left(C_{1}\right) r\left(p_{n, t}\right) \\
& =(n-1)(1)-(1)(t-1)=n-t .
\end{aligned}
$$

Now Lemma 2.2 implies that

$$
\begin{aligned}
{\left[\begin{array}{l}
h \\
\ell
\end{array}\right] } & =M\left[\begin{array}{l}
q_{1} \\
q_{2}
\end{array}\right] \geq M\left[\begin{array}{l}
t-1 \\
n-t
\end{array}\right] \\
& =\left[\begin{array}{l}
n^{2}-3 n+1+t \\
n-1
\end{array}\right] .
\end{aligned}
$$

Therefore $k_{v_{n}, v_{1}}\left(v_{t}\right) \geq n^{2}-2 n+t$ for all $1 \leq t \leq n$. Since $t \geq 1$, we conclude that $k_{v_{n}, v_{1}}\left(W_{n}^{(2)}\right) \geq n^{2}-2 n+1$ and by (2) we have $k\left(W_{n}^{(2)}\right) \geq n^{2}-2 n+1$.

We next show that $k\left(W_{n}^{(2)}\right) \leq n^{2}-2 n+1$. We show that for each $t=1,2, \ldots, n$, there is a $v_{t} \stackrel{(h, \ell)}{\longrightarrow} v_{1}$ walk with

$$
\left[\begin{array}{l}
h \\
\ell
\end{array}\right]=\left[\begin{array}{l}
n^{2}-3 n+2 \\
n-1
\end{array}\right] .
$$

By Proposition 2.1 it suffices to show that the system of equation

$$
M \mathbf{z}+\left[\begin{array}{l}
r\left(p_{t, 1}\right) \\
b\left(p_{t, 1}\right)
\end{array}\right]=\left[\begin{array}{l}
n^{2}-3 n+2 \\
n-1
\end{array}\right]
$$

has a nonnegative integer solution for some path $p_{t, 1}$ from $v_{t}$ to $v_{1}$.

The solution to the system (9) is the integer vector

$$
\mathbf{z}=\left[\begin{array}{l}
(n-2) b\left(p_{t, 1}\right)-r\left(p_{t, 1}\right) \\
n-1+r\left(p_{t, 1}\right)+b\left(p_{t, 1}\right)-b\left(p_{t, 1}\right) n
\end{array}\right] .
$$

If $1 \leq t \leq n-1$, then there is a $(n-1-t, 1)$-path $p_{t, 1}$ from $v_{t}$ to $v_{1}$. Using this path we find that $z_{1}=t-1$ and $z_{2}=n-1-t$. Since $t \geq 1$ we have $z_{1} \geq 0$ and since $t \leq n-1$ we have $z_{2} \geq 0$.

If $t=n$, there is a $(0,1)$-path $p_{n, 1}$ from $v_{n}$ to $v_{1}$. Using this path we have $z_{1}=n-2$ and $z_{2}=0$.

Thus for each $t=1,2, \ldots, n$, there is a path $p_{t 1}$ from $v_{t}$ to $v_{1}$ such that the system (9) has nonnegative integer solution. 
By Proposition 2.1 for each vertex $v_{t}, t=1,2, \ldots, n$, there is an $(h, \ell)$-walk from $v_{t}$ to $v_{1}$ with $h=n^{2}-3 n+2$ and $\ell=n-1$. Therefore, for each pair of distinct vertices $v_{i}$ and $v_{j}$ there is vertex $v_{1}$ with the property that there exist $v_{i} \stackrel{(h, \ell)}{\longrightarrow} v_{1}$ walk and $v_{j} \stackrel{(h, \ell)}{\longrightarrow} v_{1}$ walk with

$$
\left[\begin{array}{l}
h \\
\ell
\end{array}\right]=\left[\begin{array}{l}
n^{2}-3 n+2 \\
n-1
\end{array}\right]
$$

Therefore, we conclude that $k\left(W_{n}^{(2)}\right) \leq n^{2}-2 n+1$.

The following theorem presents the scrambling index of primitive two-colored Wieland digraph with two blue arcs that have the same initial vertex.

Theorem 3.4 Let $W_{n}^{(2)}$ be a two colored Wielandt digraph on $n \geq 4$ vertices. If $W_{n}^{(2)}$ has two blue arcs $v_{n-1} \rightarrow v_{1}$ and $v_{n-1} \rightarrow v_{n}$, then $k\left(W_{n}^{(2)}\right)=n^{2}-2 n+2$.

Proof. We show that $k\left(W_{n}^{(2)}\right) \geq n^{2}-2 n+2$. It suffices to show that $k_{v_{n-1}, v_{n}}\left(W_{n}^{(2)}\right) \geq n^{2}-2 n+2$. For this purpose we assume that there are $v_{n} \stackrel{(h, \ell)}{\longrightarrow} w$ and $v_{n-1} \stackrel{(h, \ell)}{\longrightarrow} w$ for some $w \in W_{n}^{(2)}$. We set up a lower bound fro $k_{v_{n-1}, v_{n}}(w)$ and consider two cases depending on the position of the vertex $w$.

Case 1. The vertex $w=v_{t}$ where $1 \leq t \leq n-1$

There is a unique path $p_{n, t}$ from $v_{n}$ to $v_{t}$ which is a $(t, 0)$ path. Using this path and (4) we have

$$
\begin{aligned}
q_{1} & \geq b\left(C_{2}\right) r\left(p_{n, t}\right)-r\left(C_{2}\right) b\left(p_{n, t}\right) \\
& =(1)(t)-(n-2)(0)=t .
\end{aligned}
$$

There are two $p_{n-1, t}$ paths from $v_{n-1}$ to $v_{t}$. They are a $(t-$ $1,1)$-path and a $(t, 1)$-path. Considering the $(t-1,1)$-path and (5) we have

$$
\begin{aligned}
q_{2} & \geq r\left(C_{1}\right) b\left(p_{n-1, t}\right)-b\left(C_{1}\right) r\left(p_{n-1, t}\right) \\
& =(n-1)(1)-(1)(t-1)=n-t .
\end{aligned}
$$

Considering the $(t, 1)$-path and (5) we have

$$
\begin{aligned}
q_{2} & \geq r\left(C_{1}\right) b\left(p_{n-1, t}\right)-b\left(C_{1}\right) r\left(p_{n-1, t}\right) \\
& =(n-1)(1)-(1)(t)=n-t-1
\end{aligned}
$$

Hence we conclude that $q_{2} \geq n-t-1$.

Now Lemma 2.2 implies that

$$
\begin{aligned}
{\left[\begin{array}{l}
h \\
\ell
\end{array}\right] } & =M\left[\begin{array}{l}
q_{1} \\
q_{2}
\end{array}\right] \geq M\left[\begin{array}{l}
t \\
n-t-1
\end{array}\right] \\
& =\left[\begin{array}{l}
n^{2}-3 n+2+t \\
n-1
\end{array}\right] .
\end{aligned}
$$

Thus

$$
k_{v_{n-1}, v_{n}}\left(v_{t}\right) \geq n^{2}-2 n+1+t
$$

for all $1 \leq t \leq n-1$.

Case 2. The vertex $w=v_{n}$

There is a $(n-1,1)$-path from $v_{n}$ to $v_{n}$. Using this path and (4) we find that

$$
\begin{aligned}
q_{1} & \geq b\left(C_{2}\right) r\left(p_{n, n}\right)-r\left(C_{2}\right) b\left(p_{n, n}\right) \\
& =(1)(n-1)-(n-2)(1)=1 .
\end{aligned}
$$

There is a $(0,1)$-path from $v_{n-1}$ to $v_{n}$. Using this path and (5) we find that

$$
\begin{aligned}
q_{2} & \geq r\left(C_{1}\right) b\left(p_{n-1, n}\right)-b\left(C_{1}\right) r\left(p_{n-1, n}\right) \\
& =(n-1)(1)-(1)(0)=n-1 .
\end{aligned}
$$

Now Lemma 2.2 implies that

$$
\begin{aligned}
{\left[\begin{array}{l}
h \\
\ell
\end{array}\right] } & =M\left[\begin{array}{l}
q_{1} \\
q_{2}
\end{array}\right] \geq M\left[\begin{array}{l}
1 \\
n-1
\end{array}\right] \\
& =\left[\begin{array}{l}
n^{2}-2 n+1 \\
n
\end{array}\right] .
\end{aligned}
$$

Thus

$$
k_{v_{n-1}, v_{n}}\left(v_{n}\right) \geq n^{2}-n+1 .
$$

By considering (10) and (11) we conclude that $k_{v_{n-1}, v_{n}}\left(W_{n}^{(2)}\right) \geq n^{2}-2 n+2$ and by (2) we conclude $k\left(W_{n}^{(2)}\right) \geq n^{2}-2 n+2$.

We next show that $k\left(W_{n}^{(2)}\right) \leq n^{2}-2 n+2$. For each vertex $v_{t}, t=1,2, \ldots, n$, we show that there is $v_{t} \stackrel{(h, \ell)}{\longrightarrow} v_{1}$ walk with

$$
\left[\begin{array}{l}
h \\
\ell
\end{array}\right]=\left[\begin{array}{l}
n^{2}-3 n+3 \\
n-1
\end{array}\right] .
$$

By Proposition 2.1 it suffices to show that the system of equations

$$
M \mathbf{z}+\left[\begin{array}{l}
r\left(p_{t, 1}\right) \\
b\left(p_{t, 1}\right)
\end{array}\right]=\left[\begin{array}{l}
n^{2}-3 n+3 \\
n-1
\end{array}\right]
$$

has a nonnegative integer solution for some path $p_{t, 1}$ from $v_{t}$ to $v_{1}$.

The solution to the system (12) is the integer vector

$$
\mathbf{z}=\left[\begin{array}{l}
1+(n-2) b\left(p_{t, 1}\right)-r\left(p_{t, 1}\right) \\
n-2+r\left(p_{t, 1}\right)+b\left(p_{t, 1}\right)-b\left(p_{t, 1}\right) n
\end{array}\right] .
$$

If $1 \leq t \leq n-1$, then there is a $(n-t, 1)$-path $p_{t, 1}$ from $v_{t}$ to $v_{1}$. Using this path we find $z_{1}=t-1$ and $z_{2}=n-1-t$. Since $t \geq 1$ we have $z_{1} \geq 0$, and since $t \leq n-1$ we have $z_{2} \geq 0$.

If $t=n$, then there is a $(1,0)$-path $p_{n, 1}$ from $v_{n}$ to $v_{1}$. Using this path we have $z_{1}=0$ and $z_{2}=n-1$.

Therefore, for each $t=1,2, \ldots, n$, the system (12) has a nonnegative integer solution for some path $p_{t, 1}$ from $v_{t}$ to $v_{1}$. By Proposition 2.1 for each vertex $v_{t}, t=1,2, \ldots, n$, there is an $(h, \ell)$-walk from $v_{t}$ to $v_{1}$ with $h=n^{2}-3 n+3$ and $\ell=n-1$. We now conclude for each pair of distinct vertices $v_{i}$ and $v_{j}$ there is vertex $v_{1}$ with the property that there are $v_{i} \stackrel{(h, \ell)}{\longrightarrow} v_{1}$ and $v_{j} \stackrel{(h, \ell)}{\longrightarrow} v_{1}$ walks with

$$
\left[\begin{array}{l}
h \\
\ell
\end{array}\right]=\left[\begin{array}{l}
n^{2}-3 n+3 \\
n-1
\end{array}\right] .
$$

Hence $k\left(W_{n}^{(2)}\right) \leq n^{2}-2 n+2$.

Let $S_{n}^{W_{n}^{(2)}}$ denote the set of positive integers $k$ for which there exists a primitive two-colored Wielandt digraph with scrambling index equals to $k$. The following result gives the characterization for the set $S_{n}^{W_{n}^{(2)}}$.

Corollary 3.5 Let $W_{n}^{(2)}$ be a primitive two-colored Wielandt digraph on $n \geq 4$ vertices. Then $S_{n}^{W_{n}^{(2)}}=\left\{k: n^{2}-3 n+3 \leq\right.$ $\left.k \leq n^{2}-2 n+2\right\}$. 
Proof. We note from Theorem 3.2 that $\left[n^{2}-3 n+3, n^{2}-\right.$ $2 n] \subseteq S_{n}^{W_{n}^{(2)}}$ since $1 \leq a \leq n-2$. By Theorem 3.3 and Theorem 3.4 we conclude that $\left[n^{2}-3 n+3, n^{2}-2 n+2\right] \subseteq$ $S_{n}^{W_{n}^{(2)}}$. Since there are only $n$ distinct primitive two-colored Wielandt digraphs on $n$ vertices, we have $S_{n}^{W_{n}^{(2)}}=\left[n^{2}-\right.$ $\left.3 n+3, n^{2}-2 n+2\right]$.

\section{REFERENCES}

[1] M. Akelbek, S. Kirkland. Coefficients of ergodicity and the scrambling index, Linear Algebra and its Applications, 430, 1111-1130, 2009.

[2] M. Akelbek, S. Kirkland. Primitive digraphs with the largest scrambling index, Linear Algebra and its Applications, 430,
1099-1110, 2009.

[3] D.S. Ananichev, M. V. Volkov and V. V. Gusev, Primitive Digraphs with Large Exponents and Slowly Synchronizing Automata, Journal of Mathematical Sciences, Vol. 192 No. 3 (2013), 263-278

[4] R. A. Brualdi and H. J. Ryser, Combinatorial Matrix Theory, Cambridge University Press, 1991.

[5] E. Fornasini, M. E. Valcher. Primitivity positive matrix pairs: algebraic characterization graph theoritic description and 2D systems interpretations, SIAM J. Matrix Anal. Appl., 19, 7188, 1998.

[6] B. L. Shader, S. Suwilo, Exponents of nonnegative matrix pairs. Linear Algebra and its Applications, 263, 275-293, 2003. 